\title{
Majorization properties of generalized thermal distributions
}

\author{
N. Canosa*, R. Rossignoli, M. Portesi \\ Departamento de Física, Facultad de Ciencias Exactas, Universidad Nacional de La Plata, C.C.67, La Plata (1900), Argentina
}

Available online 9 January 2006

\begin{abstract}
We examine the majorization properties of general thermal-like mixed states depending on a set of parameters. Sufficient conditions which ensure the increase in mixedness, and hence of any associated entropic form, when these parameters are varied, are identified. We then discuss those exhibiting a power law distribution, showing that they can be characterized by two distinct mixing parameters, one associated with temperature and the other with the non-extensivity index $q$. Illustrative numerical results are also provided.
\end{abstract}

(C) 2006 Elsevier B.V. All rights reserved.

Keywords: Generalized thermal states; Mixedness; Majorization

The rigorous concept of disorder derived from the theory of majorization [1-4] has recently received renewed attention in theoretical physics, particularly in the field of quantum information [5-7]. The essential reason is that it is stronger than that based on standard entropic considerations. The basic idea is that a given probability distribution or density matrix can be said to be "more mixed" or "disordered" than another only when it is majorized by the latter. This implies a higher entropy of the former, although the converse implication is not necessarily true. Majorization provides a natural partial ordering on probability distributions [2] and has consequently found many applications not only in mathematics, but also in other areas such as economy and computer science. Moreover, majorization relations are often naturally satisfied. For instance, in discrete classical systems, the joint distribution of two random variables is always majorized by the marginal distributions, while in quantum mechanics, the global density matrix $\rho$ of a separable (i.e., non-entangled) mixed state of a composite system is always more mixed than the local reduced densities $\rho_{i}$ of each subsystem [7], a property which can be violated by entangled states. These statements are stronger than the corresponding entropic inequalities (i.e., $S(\rho)>S\left(\rho_{i}\right)$ ).

The aim of this work is to examine along the previous lines the majorization properties of general thermallike mixed states depending on a set of parameters, discussing as application those characterized by a powerlaw distribution [8-10]. The latter have in recent years been analyzed and successfully employed in a wide range of contexts [11-15], and can be derived within a generalized non-extensive thermodynamic formalism based on the Tsallis entropy [8]. One of the basic physical questions we want to answer is if such states do become more disordered, in the way determined by majorization, when the temperature (or some other

\footnotetext{
*Corresponding author.

E-mail address: canosa@fisica.unlp.edu.ar (N. Canosa).
} 
fundamental parameter characterizing the distribution) is increased, as occurs with the standard Boltzmann-Gibbs thermal state. Such property would have far reaching consequences, in particular that of ensuring a universal entropy increase, i.e., an increase in any consistent disorder measure, and not just in that employed in the construction of the state. It would also imply the increase of the expectation value of any increasing function of energy, and not just of the energy itself. Here we will prove that such property is indeed valid.

For this purpose, we first identify the sufficient conditions that ensure the increase in mixedness of a general thermal-like mixed state when the parameters that characterize it are varied. We then show that states exhibiting a power-law distribution can be characterized by two distinct mixing parameters, one associated with temperature and the other with the non-additivity index $q$. We also discuss the majorization properties of escort distributions and the mixing conditions for generalized thermal-like states in the presence of constraints on non-commuting observables. Illustrative numerical results for a simple model are provided as well. Distributions with correct mixing properties can then be employed to investigate the effects of randomness in physical systems in a more complete way than that provided by the standard thermal state, allowing to explore different paths from the ground state to the full random state, all characterized by a proper disorder increase.

We will consider a quantum system of finite dimension $n$. The eigenvalues $p_{i}$ of any density matrix $\rho$ for such system $\left(p_{i} \geqslant 0, \sum_{i=1}^{n} p_{i}=1\right)$ will be sorted in what follows in decreasing order $\left(p_{i} \geqslant p_{j}\right.$ if $(i<j)$. A density $\rho$ is then said to be more mixed than a second density $\rho^{\prime}\left(\rho \prec \rho^{\prime}\right)$ if the eigenvalues of $\rho$ are majorized by those of $\rho^{\prime}$ [2-4]:

$$
\rho \prec \rho^{\prime} \Leftrightarrow s_{j} \equiv \sum_{i=1}^{j} p_{i} \leqslant s_{j}^{\prime} \equiv \sum_{i=1}^{j} p_{i}^{\prime}, \quad j=1, \ldots, n-1,
$$

with $s_{n}=s_{n}^{\prime}=1$. In such a case, the probabilities $p_{i}$ are more "spread out" than the $p_{i}^{\prime}$ 's, and can be written as a convex combination of permutations of the latter, i.e., $\mathbf{p}=\sum_{\alpha} q_{\alpha} P_{\alpha}\left(\mathbf{p}^{\prime}\right)$, where $P_{\alpha}$ are permutations and $q_{\alpha} \geqslant 0, \sum_{\alpha} q_{\alpha}=1$ [2-4]. The state described by $\rho$ is then more mixed or "random" than that described by $\rho^{\prime}$. Accordingly, the completely random state $\rho=I / n$ (with $I$ the identity) is more mixed than any density, while any density is more mixed than a pure state, i.e., $I / n \prec \rho \prec|\Phi\rangle\langle\Phi| \forall$ normalized density $\rho$ and pure state $|\Phi\rangle$. It can be also shown that if $\rho \prec \rho^{\prime}, \rho$ can be written as a convex combination of unitary transformations of $\rho^{\prime}$, i.e., $\rho=\sum_{\alpha} q_{\alpha} U_{\alpha}^{\dagger} \rho^{\prime} U_{\alpha}$, with $q_{\alpha}>0$ and $U_{\alpha}^{\dagger} U_{\alpha}=I$, and viceversa (Uhlmann's theorem [3]). If the dimensions of $\rho$ and $\rho^{\prime}$ differ, the same definition (1) can be applied after completing with zeros the set of eigenvalues of the density of lowest dimension.

Let us briefly discuss now the relation with entropy. Consider for instance the general entropic forms [16-19]

$$
S_{f}(\rho)=\operatorname{Tr} f(\rho)=\sum_{i=1}^{n} f\left(p_{i}\right),
$$

where $f$ is a smooth strictly concave function $\left(f^{\prime}\left(p_{i}\right)<f^{\prime}\left(p_{j}\right)\right.$ if $\left.p_{i}>p_{j}\right)$ defined in the interval $[0,1]$, satisfying $f(0)=f(1)=0$. The von Neumann entropy $S(\rho)=-\operatorname{Tr} \rho \ln \rho$ and the Tsallis generalization [8],

$$
S_{q}(\rho)=\operatorname{Tr}\left(\rho-\rho^{q}\right) /(q-1), \quad q>0,
$$

which approaches the von Neumann entropy for $q \rightarrow 1$, are the most important examples. It can be shown that if $\rho \prec \rho^{\prime} \Rightarrow S_{f}(\rho) \geqslant S_{f}\left(\rho^{\prime}\right)$ for any $f$ of the previous form [3,20] (the same holds for the Renyi entropy $S_{q}^{R}=\ln \left[1+(1-q) S_{q}(\rho)\right] /(1-q)$ [21], since it is an increasing function of $S_{q}(\rho)$, as well as for any Schur concave function of $\rho$ [4]). However, for a given $f$, the converse is not necessarily true, so that the concept of disorder implied by Eq. (1) is stronger than that based on a particular choice of $f$. Nonetheless, the converse holds as follows: if $S_{f}(\rho) \geqslant S_{f}\left(\rho^{\prime}\right)$ for any $f$ of the previous form $\Rightarrow \rho \prec \rho^{\prime}[3,20]$. In other words, the hallmark of increasing mixedness is a universal entropy increase. Note, however, that Eqs. (1) define a partial order relationship, in the sense that given two densities $\rho, \rho^{\prime}$, it may happen that $\rho \nprec \rho^{\prime}$ and $\rho^{\prime} \nprec \rho$. 
Let us now consider a general mixed state $\rho(\lambda)$ depending on a continuous parameter $\lambda$. We will say that $\lambda$ is a mixing parameter in a certain interval if $\rho(\lambda)$ becomes more mixed as $\lambda$ increases in this interval:

$$
\rho(\lambda) \prec \rho\left(\lambda^{\prime}\right) \text { if } \lambda \geqslant \lambda^{\prime} .
$$

This is equivalent, in the case of a smooth dependence, to the condition

$$
\partial s_{j} / \partial \lambda \leqslant 0, \quad j=1, \ldots, n-1,
$$

within this interval. The generalized entropy $S_{f}[\rho(\lambda)]$ is then a non-decreasing function of $\lambda$ for any concave $f$, as easily verified:

$$
\frac{\partial S_{f}[\rho(\lambda)]}{\partial \lambda}=\sum_{j=1}^{n-1} \frac{\partial s_{j}}{\partial \lambda}\left[f^{\prime}\left(p_{j}\right)-f^{\prime}\left(p_{j+1}\right)\right] \geqslant 0,
$$

since $f^{\prime}\left(p_{j}\right)-f^{\prime}\left(p_{j+1}\right) \leqslant 0$ for $f$ concave. Such states exhibit then an unambiguous disorder increase for increasing $\lambda$.

As a general example, let us consider the escort densities [21]

$$
\rho_{q}=\rho^{q} / Z_{q}, \quad Z_{q}=\operatorname{Tr} \rho^{q},
$$

associated with a density matrix $\rho$. It is easily seen that for $q>0, \lambda=1 / q$ is a mixing parameter for $\rho_{q}$, since the ensuing partial sums satisfy, for $j<n$,

$$
\frac{\partial s_{j}}{\partial \lambda}=-q^{2} \sum_{i=1}^{j} \sum_{k=j+1}^{n} p_{q i} p_{q j} \ln \left(p_{i} / p_{j}\right) \leqslant 0, \quad \lambda=1 / q>0,
$$

where $p_{q i}=p_{i}^{q} / Z_{q}$ are the eigenvalues of $\rho_{q}\left(p_{q i} \geqslant p_{q j} \forall q>0\right.$ if $\left.i \leqslant j\right)$. Any other decreasing function of $q$ is of course a mixing parameter for $\rho_{q}$ as well. We have therefore $\rho_{q} \prec \rho_{q^{\prime}}$ if $0<q \leqslant q^{\prime}$ for a given fixed density matrix $\rho$.

Assume now that the system is described by a Hamiltonian $H$ with energies $\varepsilon_{i}, i=1, \ldots, n$, sorted in what follows in increasing order, and consider densities $\rho(\lambda)$ which satisfy the conditions: (a) they commute with $H$, (b) their eigenvalues are non-increasing functions of energy $\left(p_{i} \geqslant p_{j}\right.$ if $\left.\varepsilon_{i} \leqslant \varepsilon_{j}\right)$ and (c) $\lambda$ is a mixing parameter for $\rho(\lambda)$ in a certain interval. In such a case, another fundamental consequence of Eq. (4) is that not only the average energy $\langle H\rangle_{\rho}=\operatorname{Tr} \rho(\lambda) H$, but also the expectation value of any non-decreasing function $w$ of $H$ $\left(w\left(\varepsilon_{i}\right) \leqslant w\left(\varepsilon_{j}\right)\right.$ if $\left.i<j\right)$, independent of $\lambda$, is a non-decreasing function of $\lambda$ :

$$
\frac{\partial\langle w(H)\rangle_{\rho}}{\partial \lambda}=\sum_{j=1}^{n-1} \frac{\partial s_{j}}{\partial \lambda}\left[w\left(\varepsilon_{j}\right)-w\left(\varepsilon_{j+1}\right)\right] \geqslant 0 .
$$

This automatically ensures a non-negative generalized "specific heat" $c_{\lambda} \equiv \partial\langle H\rangle_{\rho} / \partial \lambda \geqslant 0$. Let us also remark that if $\partial s_{j} / \partial \lambda$ were positive for some $j$ (and $p_{j}>p_{j+1}, \varepsilon_{j}<\varepsilon_{j+1}$ ), one could always find functions $f$ and $w$ of the previous forms such that Eqs. (5) and (8) become negative. In this way, one can in principle always witness the absence of proper mixing increase.

We may now say that $\rho(\lambda)$ exhibits a thermal-like behavior if in addition, it approaches the ground state density $I_{1} / n_{1}$ in some limit $\lambda \rightarrow \lambda_{0}\left(I_{1}\right.$ denotes the projector onto the ground state energy subspace and $n_{1}$ its degeneracy) and the state of maximum disorder $I / n$ in some other limit $\lambda \rightarrow \lambda_{\infty}$, with $\lambda$ a mixing parameter for $\lambda_{0}<\lambda<\lambda_{\infty}$. The most common example of a state of the previous form is, of course, the standard Boltzmann-Gibbs (BG) thermal state (we set in what follows Boltzmann constant $k=1$ )

$$
\rho(T)=\exp [-H / T] / Z(T), \quad Z(T)=\operatorname{Tr} \exp [-H / T], \quad T>0,
$$

obtained from the minimization of $\langle H\rangle_{\rho}-T S(\rho)$. It is well known that its von Neumann entropy $S[\rho(T)]$ is an increasing function of temperature, which is usually taken as the basis for the statement that $\rho(T)$ becomes more disordered as $T$ increases. However, in the present framework this statement can be more rigorously formulated. It is easily shown that $T$ is a proper mixing parameter for $\rho(T)$ in the interval $(0, \infty)$, i.e.,

$$
\rho(T) \prec \rho\left(T^{\prime}\right) \text { if } \quad T \geqslant T^{\prime}>0,
$$


as the sums of its first $j$ eigenvalues satisfy, for $j=1, \ldots, n-1$,

$$
\frac{\partial s_{j}}{\partial T}=\sum_{i=1}^{j} \sum_{k=j+1}^{n} p_{i} p_{k}\left(\varepsilon_{i}-\varepsilon_{k}\right) / T^{2} \leqslant 0 .
$$

Hence, not only its von Neumann entropy, but also its generalized entropy $S_{f}[\rho(T)]$, is an increasing function of $T$ for any concave $f$. The average of any increasing function of energy is an increasing function of $T$ as well. It can be shown that the generalized thermal density obtained from the minimization of $\langle H\rangle_{\rho}-T S_{f}(\rho)$, given by [18] $\rho_{f}(T)=f^{\prime-1}(H / T+\alpha)$, where $\alpha$ is a normalization constant and the cutoff $f^{\prime-1}(u)=0$ if $u>f^{\prime}(0)$ applies, also becomes more mixed as $T$ increases [15]. Eq. (11) remains valid replacing $p_{i} p_{k}$ by $\tilde{Z} \tilde{p}_{i} \tilde{p}_{k}$, with $\tilde{p}_{i}=$ $-\left[f^{\prime \prime}\left(p_{i}\right) \tilde{Z}\right]^{-1}$ for $p_{i}>0$ and $\tilde{Z}=-\sum_{p_{i}>0}\left[f^{\prime \prime}\left(p_{i}\right)\right]^{-1}$, being both positive for $f$ concave.

Let us now identify the conditions which ensure that $\lambda$ is a mixing parameter for a density of the more general form

$$
\rho(\lambda)=g(H, \lambda) / Z(\lambda), \quad Z(\lambda)=\operatorname{Tr} g(H, \lambda),
$$

where $g(\varepsilon, \lambda)$ is assumed to be an arbitrary smooth positive non-increasing function of $\varepsilon$ for $\varepsilon \in\left[\varepsilon_{1}, \varepsilon_{n}\right]$ depending on a parameter $\lambda$, with $[\rho(\lambda), H]=0$. The variation rate of the associated partial sums can be shown to be, for $j<n$,

$$
\frac{\partial s_{j}}{\partial \lambda}=\sum_{i=1}^{j} \sum_{k=j+1}^{n} p_{i} p_{k}\left[\tilde{g}_{\lambda}\left(\varepsilon_{i}\right)-\tilde{g}_{\lambda}\left(\varepsilon_{k}\right)\right], \quad \tilde{g}_{\lambda}(\varepsilon) \equiv \frac{\partial \ln g}{\partial \lambda},
$$

with $p_{i}=g\left(\varepsilon_{i}, \lambda\right) / Z(\lambda)$, which generalizes Eq. (11). A sufficient condition which ensures $\partial s_{j} / \partial \lambda \leqslant 0$ for $j=$ $1, \ldots, n-1$ is, therefore, that $\tilde{g}_{\lambda}(\varepsilon)$ be a non-decreasing function of $\varepsilon$ for $\varepsilon \in\left[\varepsilon_{1}, \varepsilon_{n}\right]$, i.e.,

$$
\frac{\partial^{2} \ln g}{\partial \varepsilon \partial \lambda} \geqslant 0 \Rightarrow \frac{\partial s_{j}}{\partial \lambda} \leqslant 0, \quad j=1, \ldots, n-1 .
$$

For instance, if

$$
\rho(\lambda)=g(H / \lambda) / Z(\lambda)
$$

where $\lambda>0$ and $g$ is here a positive non-increasing smooth real function, $\tilde{g}_{\lambda}(\varepsilon)=-\lambda^{-1} u[\ln g(u)]^{\prime}$, with $u=\varepsilon / \lambda$, and Eq. (14) leads to the condition

$$
-\left\{u[\ln g(u)]^{\prime}\right\}^{\prime} \geqslant 0 \text {, }
$$

which is not necessarily valid (consider for instance $g(u)=[1+\ln (u+1)]^{-1}$ for $u>0$ ). It is, of course, valid in the BG case for $\lambda=T(g(u)=\exp [-u])$ as well as for $g(u)=\exp \left(-u^{r}\right) \forall r>0$ if $u>0\left(-\left\{u[\ln g(u)]^{\prime}\right\}^{\prime}=\right.$ $\left.r^{2} u^{r-1} \geqslant 0\right)$. Note also that if $g(0)>0$ and $g(\infty)=0$, the state $\rho(\lambda)=g(\bar{H} / \lambda) / Z(\lambda)$, with $\bar{H}=H-\varepsilon_{1} I$, will always approach the ground state density $I_{1} / n_{1}$ for $\lambda \rightarrow 0$ and the fully mixed state $I / n$ for $\lambda \rightarrow \infty$. It is, however, the more stringent condition (16) which ensures that $\rho(\lambda)$ will in addition become monotonously more mixed as it evolves from the ground state to the fully mixed state.

Note also that if $\lambda$ is a mixing parameter for the density (12), it will remain a mixing parameter for the associated escort density (6) for $q>0$, which corresponds to $g_{q}(H, \lambda)=g(H, \lambda)^{q}$. The sign of $\partial^{2} \ln g_{q} / \partial \varepsilon \partial \lambda$ is left unchanged for $\lambda$ independent of $q$. Eq. (7) also follows from Eq. (13) for $g(H, \lambda) \rightarrow g(H)^{1 / \lambda}$.

As an important example of Eq. (12), we will examine the mixing properties of density operators characterized by a power-law distribution, which can be written in the form of the Tsallis distribution $[8,9]$

$$
\rho\left(q, T^{*}\right)=\left[I-(1-q) \bar{H} / T^{*}\right]_{+}^{1 /(1-q)} / Z\left(q, T^{*}\right),
$$

where $T^{*}>0$ represents an effective temperature, $q$ the non-extensivity index, $\bar{H}=H-\varepsilon_{1} I$ the energy measured from the ground state and $[u]_{+} \equiv(u+|u|) / 2$. For $q \rightarrow 1, \rho(q, T)$ approaches the BG distribution (9). Eq. (17) is obviously positive and fulfills previous conditions (a) and (b) $\forall T^{*}>0$ and $q \in \mathfrak{R}$. Its eigenvalues $p_{i}$ are strictly decreasing functions of energy for $q>1$, but just non-increasing functions for $q<1$ due to the cutoff that applies in this case $\left(p_{i}=0\right.$ if $(1-q) \bar{\varepsilon}_{i} \geqslant T^{*}$, where $\left.\overline{\varepsilon_{i}} \equiv \varepsilon_{i}-\varepsilon_{1}\right)$. 
We will now show that both $T^{*}$ and $q$ are proper mixing parameters for $\rho\left(q, T^{*}\right)$. Defining $g\left(\bar{\varepsilon}, q, T^{*}\right) \equiv$ $\left[1-(1-q) \bar{\varepsilon} / T^{*}\right]_{+}^{1 /(1-q)}$, we obtain

$$
\frac{\partial^{2} \ln g}{\partial \bar{\varepsilon} \partial T^{*}}=\frac{1}{\left(T^{*}-(1-q) \bar{\varepsilon}\right)^{2}} \geqslant 0, \quad \frac{\partial^{2} \ln g}{\partial \bar{\varepsilon} \partial q}=\frac{\bar{\varepsilon}}{\left(T^{*}-(1-q) \bar{\varepsilon}\right)^{2}} \geqslant 0,
$$

for $\bar{\varepsilon}>0$ and $(1-q) \bar{\varepsilon} / T^{*}<1$, so that according to Eq. (14), $\rho\left(q, T^{*}\right)$ becomes more mixed as either $T^{*}$ or $q$ increases:

$$
\rho\left(q, T^{*}\right) \prec \rho\left(q, T^{*}\right) \text { if } T^{*} \geqslant T^{\prime *}>0, \quad \rho\left(q, T^{*}\right) \prec \rho\left(q^{\prime}, T^{*}\right) \text { if } q \geqslant q^{\prime} .
$$

The role of $T^{*}$ and $q$ as proper mixing parameters constitutes then another fundamental property of the distribution (17). Any entropy $S_{f}\left[\rho\left(q, T^{*}\right)\right]$ (in particular $S_{q^{\prime}}\left[\rho\left(q, T^{*}\right)\right]$ for any $q^{\prime}>0$ ) is a non-decreasing function of both $T^{*}$ and $q$ in any system, as illustrated in Fig. 1 for a truncated harmonic oscillator.

Let us remark that Eq. (17) exhibits a proper thermal-like behavior with respect to both $q$ and $T^{*}$, since in addition it approaches the ground state density $I_{1} / n_{1}$ both for $T^{*} \rightarrow 0$ at fixed $q$ and for $q \rightarrow-\infty$ at fixed $T^{*}$, and the random state $I / n$ both for $T^{*} \rightarrow \infty$ at fixed $q$ as well as for $q \rightarrow \infty$ at fixed $T^{*}$. Actually, due to the cutoff for $q<1, \rho\left(q, T^{*}\right)=I_{1} / n_{1}$ already for $T^{*}<(1-q) \Delta$ at fixed $q<1$, and $q<1-T^{*} / \Delta$ at fixed $T^{*}$, where $\Delta$ is the lowest non-zero excitation energy. Note also that $\rho\left(q, T^{*}\right)-I / n$ behaves linearly with $\bar{H}$ for large $T^{*}\left(g\left(\bar{\varepsilon}, q, T^{*}\right) \approx 1-\bar{\varepsilon} / T^{*}\right.$ for $\left.T^{*} \gg|1-q| \bar{\varepsilon}\right)$ but logarithmically for large $q\left(g\left(\bar{\varepsilon}, q, T^{*}\right) \approx 1-\ln \left[1+q \bar{\varepsilon} / T^{*}\right] /\right.$ $q$ for $q \gg \ln \left[1+q \bar{\varepsilon} / T^{*}\right]$ and $\left.q \gg 1\right)$.
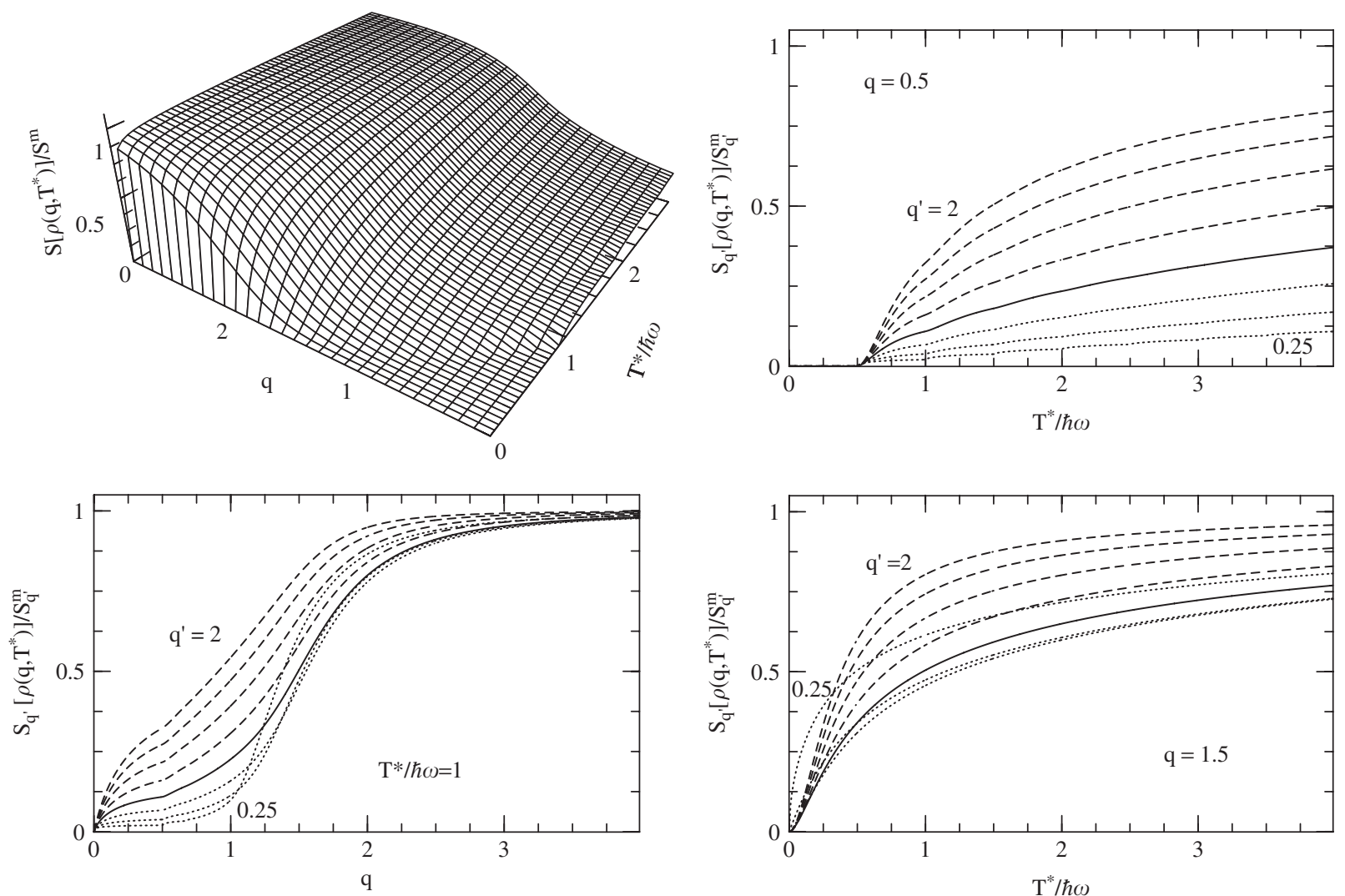

Fig. 1. Top left: the von Neumann entropy of the Tsallis distribution (17) (scaled to the maximum value $S^{m} \equiv S(I / n)$ ) for $n=100$ equally spaced levels with spacing $\hbar \omega$, showing that it is a non-decreasing function of both $T^{*}$ and $q$. Remaining panels: The scaled Tsallis entropy $S_{q^{\prime}}\left[\rho\left(q, T^{*}\right)\right] / S_{q^{\prime}}^{m}$ in the same system, as a function of $T^{*}$ at fixed $q$ (right panels) and as a function of $q$ at fixed $T^{*}$ (bottom left panel), for different values of $q^{\prime}$, ranging from $q^{\prime}=0.25$ to $q^{\prime}=2$ in steps of 0.25 . Dashed (dotted) lines correspond to $q^{\prime}>1\left(q^{\prime}<1\right)$, solid lines to $q^{\prime}=1$ (von Neumann entropy). They are all increasing functions of $T^{*}$ and $q$. 
Eq. (17) can also be characterized by other mixing parameters. For example, rewriting Eq. (17) as $\rho_{s}(\gamma, \mu)=[1-s \bar{H} / \mu]_{+}^{s / \gamma} / Z(\gamma, \mu)$, with $\gamma=|1-q|>0, \mu=T^{*} /|1-q|>0$ and $s=\operatorname{Sign}(1-q)$, both $\mu$ and $\gamma$ are as well independent mixing parameters for both signs of $s$ (despite the increase of $\gamma$ with decreasing $q$ for $q<1$ ), since $\partial^{2} \ln g / \partial \bar{\varepsilon} \partial \mu=\left[\gamma(\mu-s \bar{\varepsilon})^{2}\right]^{-1} \geqslant 0, \partial^{2} \ln g / \partial \bar{\varepsilon} \partial \gamma=\left[\gamma^{2}(\mu-s \bar{\varepsilon})\right]^{-1} \geqslant 0$ for $s \bar{\varepsilon} / \mu<1$. Thus, $\rho_{s}(\gamma, \mu) \prec \rho_{s}\left(\gamma, \mu^{\prime}\right)$ if $\mu \geqslant \mu^{\prime}$ and $\rho_{s}(\gamma, \mu) \prec \rho_{s}\left(\gamma^{\prime}, \mu\right)$ if $\gamma \geqslant \gamma^{\prime}$. These properties also follow from Eq. (16) in the case of $\mu$ (for $\left.g(u)=[1-s u]_{+}^{s / \gamma}\right)$ and from Eqs. (6)-(7) in the case of $\gamma$. Again, we obtain a thermal-like behavior with respect to both $\gamma$ and $\mu$ for $s= \pm 1$, with the ground state density approached for $\mu \rightarrow 0$ or $\gamma \rightarrow 0$ and the full random state approached for $\mu \rightarrow \infty$ or $\gamma \rightarrow \infty$ (provided $\mu>\bar{\varepsilon}_{n}$ if $s=1$ ).

The actual thermal state derived from the non-extensive thermodynamic formalism based on the Tsallis entropy and the minimization of the free energy $F_{q}=\langle H\rangle_{\rho_{q}}-T S_{q}(\rho)[10,13]$, where $q>0$ and $\rho_{q}$ is the escort density (6), is also of the form (17) but with $T^{*}$ related to the actual $T$ by $T=\left[T^{*}-(1-q)\langle\bar{H}\rangle_{\rho_{q}}\right] / Z_{q}$ (following Ref. [13]). For $q>1, T$ is a direct increasing function of $T^{*}$, as in this case $\partial T / \partial T^{*}=$ $(\eta q-1) /\left[(q-1) Z_{q}\right] \geqslant 0$ (with $\eta=2\langle O\rangle_{\rho_{q}}\left\langle O^{-1}\right\rangle_{\rho_{q}}-1 \geqslant 1$ and $O=I-(1-q) \bar{H} / T^{*}$ ), so that $T$ will also be a proper mixing parameter. It will remain so for $0<q<1$ provided the absolute minimum of the free energy $F_{q}$ at each $T$ is considered [22], as in this case the entropy (and hence $T^{*}$ ) cannot decrease with increasing $T$.

So far all previous expressions are applicable in both the quantum and the classical discrete case. Let us finally briefly examine the majorization properties of density matrices constructed from constraints on two or more non-commuting observables. We may for instance consider two observables $H_{0}, H_{1}$, with $\left[H_{0}, H_{1}\right] \neq 0$, and a density of the form

$$
\rho\left(\lambda_{0}, \lambda_{1}\right)=g\left(H_{0} / \lambda_{0}+H_{1} / \lambda_{1}\right) / Z\left(\lambda_{0}, \lambda_{1}\right)
$$

where $g(u)$ is a positive non-increasing function and $\lambda_{v}>0$ for $v=0,1$, generalizing Eq. (15). In this case, Eq. (13) should be replaced, for $j<n$, by

$$
\frac{\partial s_{j}}{\partial \lambda_{v}}=\sum_{i=1}^{j} \sum_{k=j+1}^{n} p_{i} p_{k}\left[\tilde{g}_{i}^{v}-\tilde{g}_{k}^{v}\right], \quad \tilde{g}_{i}^{v}=\frac{\partial \ln g\left(u_{i}\right)}{\partial \lambda_{v}}=-\frac{g^{\prime}\left(u_{i}\right)}{\lambda_{v}^{2} g\left(u_{i}\right)}\left\langle i\left|H_{v}\right| i\right\rangle,
$$

where $u_{i}$ denotes the eigenvalues (sorted in increasing order) of $\sum_{v=0,1} H_{v} / \lambda_{v}$ and $|i\rangle$ the corresponding eigenstates (in case of degeneracy we assume in (21) $H_{v}$ diagonal within each eigenspace). Hence, we can ensure that $\lambda_{v}$ will be a mixing parameter for (20) if $\tilde{g}_{i}^{v}$ does not decrease for increasing values of $u_{i}$.

For a single observable $H_{0}, \tilde{g}_{i}^{0}=-\lambda_{0}^{-1} u_{i} g^{\prime}\left(u_{i}\right) / g\left(u_{i}\right)$ and the previous condition reduces to Eq. (16). This is also the case when $\left\langle i\left|H_{v}\right| i\right\rangle$ is proportional to $u_{i}\left(\left\langle i\left|H_{v}\right| i\right\rangle=\alpha u_{i}\right.$, with $\alpha>0$ and independent of $\left.i\right)$, as occurs in simple systems such as a harmonic oscillator $H=\left[p^{2} / m+k x^{2}\right] / 2$. In this case $\left\langle i\left|p^{2}\right| i\right\rangle=m E_{i},\left\langle i\left|x^{2}\right| i\right\rangle=E_{i} / k$, with $E_{i}=\hbar \omega(i+1 / 2)$ the oscillator energies $(\omega=\sqrt{k / m})$, so that both the mass $m$ and the inverse oscillator strength $k^{-1}$ are also mixing parameters in $\rho=g(H / T) / Z(T)$, provided $T$ is a mixing parameter too (for $\lambda_{0}=m, \lambda_{1}=k^{-1}$, we would have $g_{i}^{v}=-(1 / 2) \lambda_{v}^{-1}\left[u_{i} g^{\prime}\left(u_{i}\right) / g\left(u_{i}\right)\right]$, with $\left.u_{i}=E_{i} / T\right)$. In the general case, however, $\left\langle i\left|H_{v}\right| i\right\rangle / u_{i}$ may depend on $i$ in a non-trivial way, so that the mixing properties of $\rho$ will require a careful analysis of the behavior of $\tilde{g}_{i}^{v}$.

In conclusion, we have applied the theory of majorization to identify the rigorous sufficient mixing conditions, as well as their main physical implications, for general mixed states of the form (12), which are summarized in Eqs. (14), (16), (5) and (8). As application, we have examined those characterized by a power law distribution, and shown that they can be expressed in terms of two fundamental mixing parameters, which can be taken as $T^{*}$ and $q$ in the representation (17). We have in particular identified the role of $q$ in (17) as a rigorous mixing parameter. The actual thermal state derived in the Tsallis non-extensive thermodynamic formalism was also shown to become more mixed for increasing $T$ (with the above remarks applying for $0<q<1$ ), as occurs with the standard BG thermal state, ensuring in particular a universal entropy increase, i.e., $\partial S_{f}[\rho(q, T)] / \partial T \geqslant 0$ for any concave $f$. These results strengthen thus the robustness of the generalized nonextensive thermodynamic formalism. We have also discussed the majorization properties of escort distributions (Eq. (6)) and derived sufficient conditions for mixedness increase in the presence of noncommuting observables (Eq. (21)). 
Majorization theory enables then to derive very general inequalities with deep implications by simple means. Generalized thermal-like distributions with proper mixing parameters may also help to provide a more complete description of the behavior of a correlated quantum system with increasing randomness, revealing aspects which could be hidden in standard BG statistics [15]. For instance, any system possessing a limit temperature $T_{c}$ in standard statistics for some property present in its ground state and absent in the vicinity of the completely random state, will also possess a limit value of the mixing parameter in a generalized thermallike distribution. Knowledge of such boundaries (like a critical curve $T_{c}^{*}(q)$ in (17)) may provide a new perspective for the classification of order-disorder transitions or crossovers.

The authors acknowledge support from CIC (RR), CONICET (NC,MP) and ANPCYT (MP) of Argentina.

\section{References}

[1] G.H. Hardy, J.E. Littlewood, G. Pólya, Inequalities, Cambridge University Press, Cambridge, 1978.

[2] A.W. Marshall, I. Olkin, Inequalities: Theory of Majorization and its Applications, Academic Press, New York, 1979.

[3] A. Wehrl, Rev. Mod. Phys. 50 (1978) 221.

[4] R. Bhatia, Matrix Analysis, Springer, Berlin, 1997.

[5] M. Nielsen, I. Chuang, Quantum Computation and Quantum Information, Cambridge University Press, Cambridge, 2000.

[6] M.A. Nielsen, Phys. Rev. Lett. 83 (1999) 436.

[7] M.A. Nielsen, J. Kempe, Phys. Rev. Lett. 86 (2001) 5184;

T. Hiroshima, Phys. Rev. Lett. 91 (2003) 057902.

[8] C. Tsallis, J. Stat. Phys. 52 (1988) 479.

[9] E.M.F. Curado, C. Tsallis, J. Phys. A 24 (1991) L69.

[10] C. Tsallis, R.S. Mendes, A.R. Plastino, Physica A 261 (1998) 534.

[11] M. Gell-Mann, C. Tsallis, Nonextensive Entropy-Interdisciplinary Applications, Oxford University Press, New York, 2004.

[12] S. Abe, Y. Okamoto, Non Extensive Statistical Mechanics and its Applications, Lecture Notes in Physics, Springer, Heidelberg, 2001.

[13] C. Tsallis, Physica D 193 (2004) 3.

[14] S. Abe, A.K. Rajagopal, Phys. Rev. Lett. 91 (2003) 120601.

[15] R. Rossignoli, N. Canosa, Phys. Lett. A 323 (2004) 22.

[16] R.S. Mendes, Physica A 242 (1997) 299.

[17] A. Plastino, A.R. Plastino, Phys. Lett. A 226 (1997) 257.

[18] R. Rossignoli, N. Canosa, Phys. Lett. A 264 (1999) 148;

N. Canosa, R. Rossignoli, Phys. Rev. Lett. 88 (2002) 170401.

[19] E.M.F. Curado, Braz. J. Phys. 29 (1999) 36.

[20] R. Rossignoli, N. Canosa, Phys. Rev. A 67 (2003) 042302;

R. Rossignoli, N. Canosa, Physica A 344 (2004) 637.

[21] C. Beck, F. Schlögl, Thermodynamic of Chaotic Systems, Cambridge University Press, Cambridge, 1993.

[22] A.R. Lima, T.J.P. Penna, Phys. Lett. A 256 (1999) 221. 